\title{
CONCEPÇÃO DE PROFISSIONAIS DA ESTRATÉGIA SAÚDE DA FAMÍLIA SOBRE VIOLÊNCIA DOMÉSTICA CONTRA A MULHER
}

\section{CONCEPTION OF PROFESSIONALS OF THE FAMILY HEALTH STRATEGY ON DOMESTIC VIOLENCE AGAINST WOMEN}

\author{
Johandra Cristina Vieira Santos, Luana Ramos da Silva, Gilberto Alves Dias, Juliana Costa \\ Machado, Vanda Palmarella Rodrigues
}

Universidade Estadual do Sudoeste da Bahia (UESB)

\begin{abstract}
Domestic violence against women has been an increasingly discussed problem that affects different areas of society linked to cultural, social and economic factors, being characterized as an important public health problem. The objective of the study was to describe the social representations of health professionals who work within the scope of the Family Health Strategy (FHS) on domestic violence against women. Descriptive qualitative research, based on the Theory of Social Representations, with 24 professionals from Family Health Units, using the software IRAMUTEq and Thematic Content Analysis. The understanding of the concept and forms of domestic violence against women among the FHS professionals were apprehended. In this sense, studies in this view identify the need for primary care professionals to better identify and report cases of domestic violence against women in their units.
\end{abstract}

Key words: Domestic Violence; Violence against Women; Health Personnel; Comprehensive Health Care.
Resumo

A violência doméstica contra a mulher tem sido um problema cada vez mais discutido que acomete diferentes âmbitos da sociedade ligados a fatores culturais, sociais e econômicos, sendo caracterizada como um importante problema de saúde pública. O objetivo do estudo foi descrever as representações sociais de profissionais de saúde que atuam no âmbito da Estratégia Saúde da Família (ESF) sobre a violência doméstica contra a mulher. Pesquisa descritiva de natureza qualitativa, embasada na Teoria das Representações Sociais, com 24 profissionais de Unidades de Saúde da Família, utilizando o software IRAMUTEq e Análise de Conteúdo Temática. Foram apreendidos o entendimento sobre o conceito $e$ as formas de violência doméstica contra a mulher entre os profissionais da ESF. Nesse sentido, estudos nessa visão demonstram a necessidade de que profissionais da atenção básica possam identificar e notificar melhor casos de violência doméstica contra a mulher em suas unidades.

Palavras chave: Violência Doméstica; Violência contra a Mulher; Pessoal de Saúde; Assistência Integral à Saúde. 
Introdução

A violência doméstica contra a mulher (VDCM) tem sido um problema cada vez mais discutido que acomete diferentes âmbitos da sociedade ligados a fatores culturais, sociais e econômicos, sendo caracterizada como um importante problema de saúde pública1. Ainda que sua ocorrência tenha registros desde a antiguidade, somente há aproximadamente 50 anos é que se observa um destaque social e político para a questão da violência, sendo acentuada a magnitude desse fenômeno enfrentado por mulheres ${ }^{2}$.

A VDCM ocorre de diferentes formas, como a violência física que é caracterizada por um ato violento que atinge diretamente o corpo e a integridade física da mulher; violência psicológica sucedida de atitudes que podem ferir a autoestima e humilhar a mulher; violência sexual por meio de qualquer ato ou tentativa de obtenção que agrida a sexualidade, independente da relação com a mulher; violência psicológica presente em palavras e xingamentos e violência patrimonial que é qualquer conduta que configure retenção, subtração, destruição parcial ou total de bens ${ }^{3}$.

De acordo com o Atlas da Violência 2019, não é possível identificar o aumento do número de feminicídios (homicídio ocorrido contra mulheres motivado por violência doméstica ou discriminação do gênero) por políticas públicas ou diminuição de subnotificações, uma vez que a Lei do Feminicídio (Lei 13.104 de 09/03/2015) é recente, assim, as autoridades judiciárias ainda se encontram em processo de conhecimento e instrução. Entretanto, notou-se que houve um aumento da taxa de homicídios contra mulheres no ano de 2017 com relação ao ano de 2007, sendo estimados 13 homicídios por dia, contabilizando ao todo 4.936 mortes desse grupo 4 .

As Unidades de Saúde da Família (USF) fazem parte dos serviços de saúde mais solicitados pelas mulheres que buscam atendimento para as marcas físicas deixadas pela agressão vivenciada, entretanto muitas delas por medo ou vergonha não fornecem informações para a notificação do agravo. Sendo assim, a VDCM é uma realidade na rotina dos profissionais de saúde que atuam na prestação dos serviços básicos em saúde ${ }^{5}$.

A notificação da violência contra a mulher deve ser realizada pelo profissional de saúde, como caráter de notificação compulsória em todo o Brasil, no entanto, observam-se dificuldades por parte dos profissionais na identificação dos casos, seja por insegurança, desatenção aos sinais de violência nas mulheres submetidas à situação ou desconhecimento das formas de violência contra a mulher. Assim, os profissionais podem desenvolver ações de prevenção, como debates com a comunidade acerca do tema, identificação, através da notificação de casos e enfrentamento da VDCM, pelo meio de ações conjuntas com a Delegacia Especializada de Atendimento à Mulher (DEAM) e comunidade 6 .

Estudar a Teoria das Representações Sociais no contexto da VDCM auxiliará a identificação e o desenvolvimento do pensamento social, uma vez que irá contribuir para a compreensão de um dado fenômeno ao analisar as relações interpessoais e a violência contra a mulher, além de compreender como o pensamento do indivíduo direcionará as condutas perante a sociedade7. Dessa forma, esse estudo traz como questão norteadora: quais as representações sociais de profissionais de saúde da Estratégia Saúde da Família (ESF) sobre a violência doméstica contra a mulher?

$\mathrm{O}$ artigo teve como objetivo geral descrever as Representações Sociais de profissionais da Estratégia Saúde da Família sobre a violência doméstica contra a mulher.

\section{Metodologia}

Pesquisa de cunho descritiva, com natureza qualitativa, embasada na Teoria das Representações Sociais (TRS) em que se estuda o pensamento social de um determinado grupo que foi construído historicamente e investiga as relações interpessoais dos mesmos ${ }^{8}$.

O cenário da pesquisa constou de cinco USF do município de Jequié, Bahia, sendo uma unidade com equipe de saúde da família única e quatro unidades com equipes duplas. Como critérios de inclusão para as USF, foram utilizados: USF nas quais funcionam duas equipes ou equipe única da ESF, localizadas na zona urbana e com equipe mínima completa, como exigem os protocolos do Ministério da Saúde. Como critérios de exclusão das USF foram selecionados: USF que apresentavam mais de duas equipes atuando na mesma estrutura física e USF que estivessem em reforma.

Entrou-se em contato com as coordenações das USF para agendamento com os profissionais de saúde para a coleta de dados. Desta forma, participaram do estudo 24 profissionais de saúde sendo, quatro enfermeiros, três técnicos de enfermagem, um auxiliar de enfermagem, 13 agentes comunitários de saúde (ACS), um 
cirurgião-dentista e dois auxiliares de saúde bucal (ASB) que atuavam em USF da zona urbana de um município do interior baiano.

Os profissionais de saúde deveriam atender os seguintes critérios: ser profissional de saúde da ESF e ter atuação mínima de seis meses na USF. Como critério de exclusão, definiu-se: estar afastado do serviço por férias ou licença de qualquer natureza.

Os dados foram coletados entre o período de agosto de 2016 a abril de 2017, utilizando uma entrevista semiestruturada. As entrevistas foram realizadas em local privativo das USF, de forma individualizada, utilizando um gravador, tendo em média cada entrevista 40 minutos. Por sua vez, a entrevista semiestruturada continha perguntas para a caracterização sociodemográfica dos profissionais de saúde e questões disparadoras sobre a violência doméstica contra a mulher (conhecimentos, atitudes, práticas).

As entrevistas foram transcritas de maneira literal para submissão à técnica de Análise de Conteúdo Lexical Lematizada com auxílio do software Iramuteq, além de trabalhar com a Análise de Conteúdo Temática de Bardin, sendo organizado em três fases: pré-análise, exploração do material e tratamento dos resultados, inferência e interpretação ${ }^{9}$. Optou-se por apresentar apenas as unidades de registro que estavam relacionadas aos achados encontrados, de modo a explicitar e ampliar a compreensão desse estudo.

Para a verificação dos dados no software Iramuteq, as falas dos profissionais de saúde foram correlacionadas de acordo às respectivas categorias profissionais. Nesse sentido, manifestou-se no banco de dados as seguintes categorias: categoria 1: agente comunitário de saúde; categoria 2: enfermeiras; categoria 3 : auxiliares de saúde bucal; categoria 4: técnicas e auxiliar de enfermagem, e categoria 5: cirurgiãodentista.

Assim, derivou-se a associação das categorias profissionais - técnica e auxiliar de enfermagem - de acordo a Portaria no 2.436 de 21 de setembro de 201710, a qual sugere à similaridade na função de ambas as categorias no contexto da ESF.

Esse estudo compõe um recorte do projeto de pesquisa: "Violência doméstica contra a mulher: representações sociais das equipes de saúde da família", o qual considerou os requisitos éticos exigidos pela Resolução 466 de 2012. Por sua vez, o projeto foi submetido ao Comitê de Ética em Pesquisa da Universidade Estadual do Sudoeste da Bahia/Campus de Jequié sob CAAE: 49736915.3.0000.0055 e aprovado sob o parecer no 1.304.618/2015.

Os participantes aceitaram colaborar com a pesquisa e assinaram o Termo de Consentimento Livre e Esclarecido (TCLE) em duas vias, uma delas ficando com a guarda das pesquisadoras. Para garantia do anonimato, os participantes foram codificados com a abreviação da categoria profissional, acompanhada de um algarismo arábico correspondente à ordem crescente de realização das entrevistas a exemplo de (ACS 1, ACS 2; ENF 1, ENF 2, etc).

\section{Resultados}

De acordo com os dados sociodemográficos dos participantes, os mesmos foram divididos em categorias profissionais para melhor descrição.

Nessa perspectiva, dos 13 ACS, 15\% eram do sexo masculino e $85 \%$ do sexo feminino, $38 \%$ tinham ensino superior completo, $23 \%$ ensino superior incompleto e $38 \%$ ensino médio completo. As idades diferenciavam-se entre as faixas de 61 a 34 anos; já o tempo de atuação profissional entre oito a 21 anos. Das quatro enfermeiras, todas eram do sexo feminino e possuíam pós-graduação, com idades entre 29 a 40 anos. O tempo de atuação profissional diferenciou entre nove meses a 10 anos.

Já, entre as quatro técnicas de enfermagem e uma auxiliar de enfermagem todas eram do sexo feminino, $75 \%$ tinham ensino médio completo e $25 \%$ ensino superior incompleto. As idades diferenciavam-se entre 38 a 47 anos e o tempo de atuação entre 13 a 25 anos. As duas auxiliares de saúde bucal eram do sexo feminino, uma com 37 anos de idade, pós-graduação e 10 anos de atuação. Já a outra tinha 45 anos de idade, ensino médio completo e 20 anos de atuação. O cirurgião-dentista era do sexo masculino, 37 anos de idade, pós-graduado com 11 anos de profissão.

Após a submissão do banco de dados ao software Iramuteq, foram criados dois planos cartesianos por meio da Análise Fatorial de Correspondência (AFC). Dessa maneira, o primeiro plano buscou descrever as representações sociais dos profissionais da ESF sobre violência doméstica contra a mulher. 
Figura 1: Plano cartesiano gerado por meio da análise das categorias profissionais.

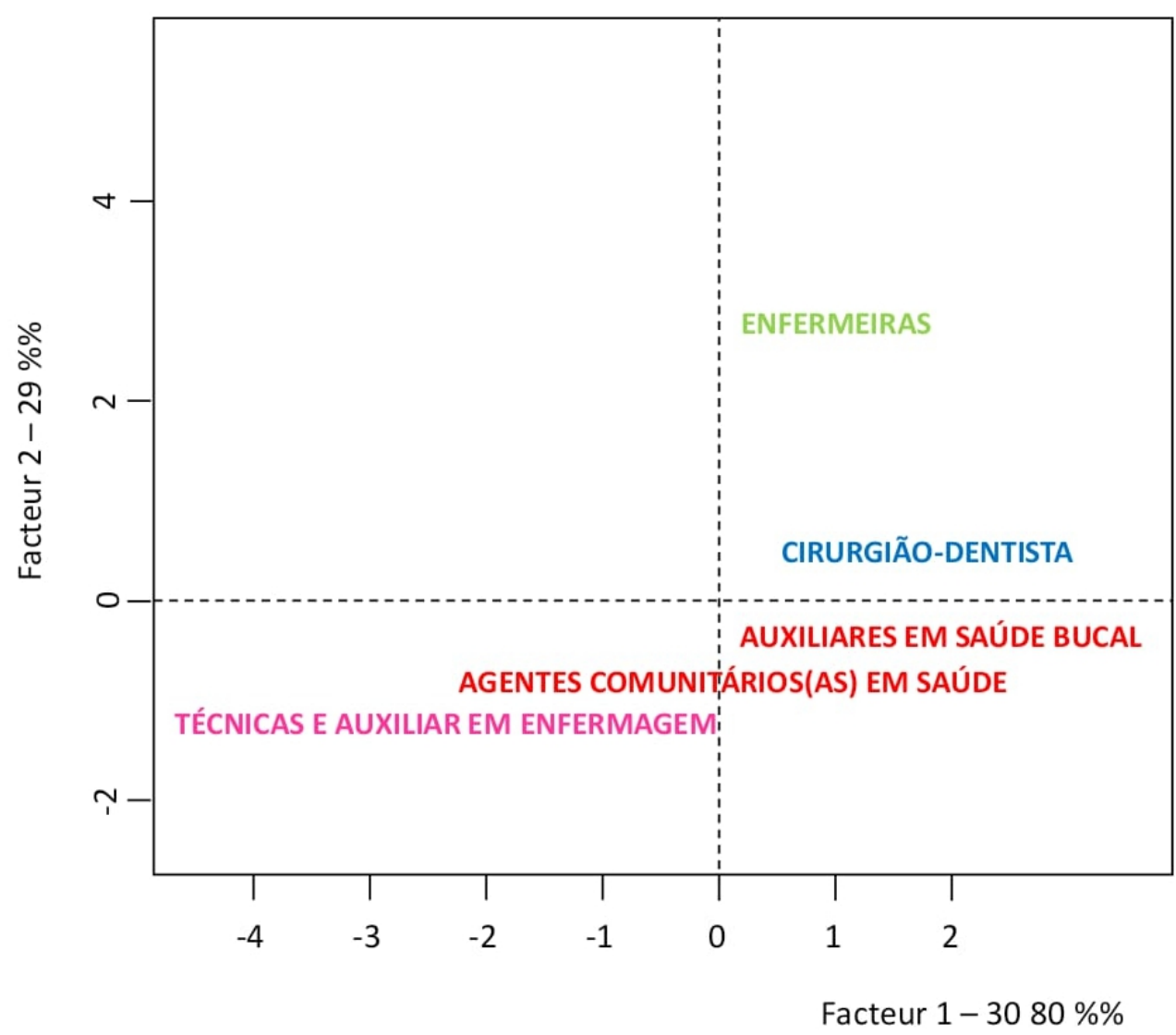

Fonte: Dados da pesquisa, 2017.

Assim, é possível observar que as cinco categorias profissionais analisadas estão próximas do eixo cartesiano. Dessa maneira, é posto que o entendimento expressado pelos participantes obtiveram forte relação, caso contrário, estariam se distanciando do eixo central do plano cartesiano. Assim, as RS dos profissionais de saúde sobre a VDCM trazem o entendimento sobre o conceito e as formas de violência doméstica contra a mulher de forma compatível.

Observa-se que algumas categorias profissionais possuem RS mais homogêneas, como os ASB, ACS e cirurgião-dentista, enquanto que o distanciamento de técnicos e auxiliares e enfermagem demonstram que este grupo social diverge $o$ pensamento social das demais categorias.

Já, a categoria de enfermeiras é apresentada com representações que mais divergem do grupo de profissionais de saúde, de acordo o quadrante superior esquerdo, o que caracteriza como sendo uma RS mais forte e a respeito do tema.
A AFC desenvolve também um segundo plano cartesiano com palavras evocadas pelos entrevistados. Assim, através do software Iramuteq foram analisadas palavras repetidas por mais de 10 vezes. Dessa forma, é demonstrado a seguir o segundo plano cartesiano.

Dessa forma, é observado que no primeiro quadrante, localizado na região superior esquerda, destacam-se as palavras: psicológico, físico e agressão. Ainda, nesse mesmo quadrante, emergem com menos ênfase, as palavras: verbal, doméstico, violência, mulher e vergonha. Essas evocações compreendem o entendimento dos ACS sobre as formas de VDCM.

Nessa perspectiva, observam-se as falas que caracterizam o primeiro quadrante:

[...] porque geralmente esses agressores fazem com que essa mulher fique com uma autoestima muito baixa, muito abalada, porque eles têm que fazer com que elas fiquem submissas a eles, então o psicológico também é muito afetado (ACS 2). 
Figura 2: Plano cartesiano gerado por meio das entrevistas das(os) profissionais de saúde da Estratégia de Saúde da Família

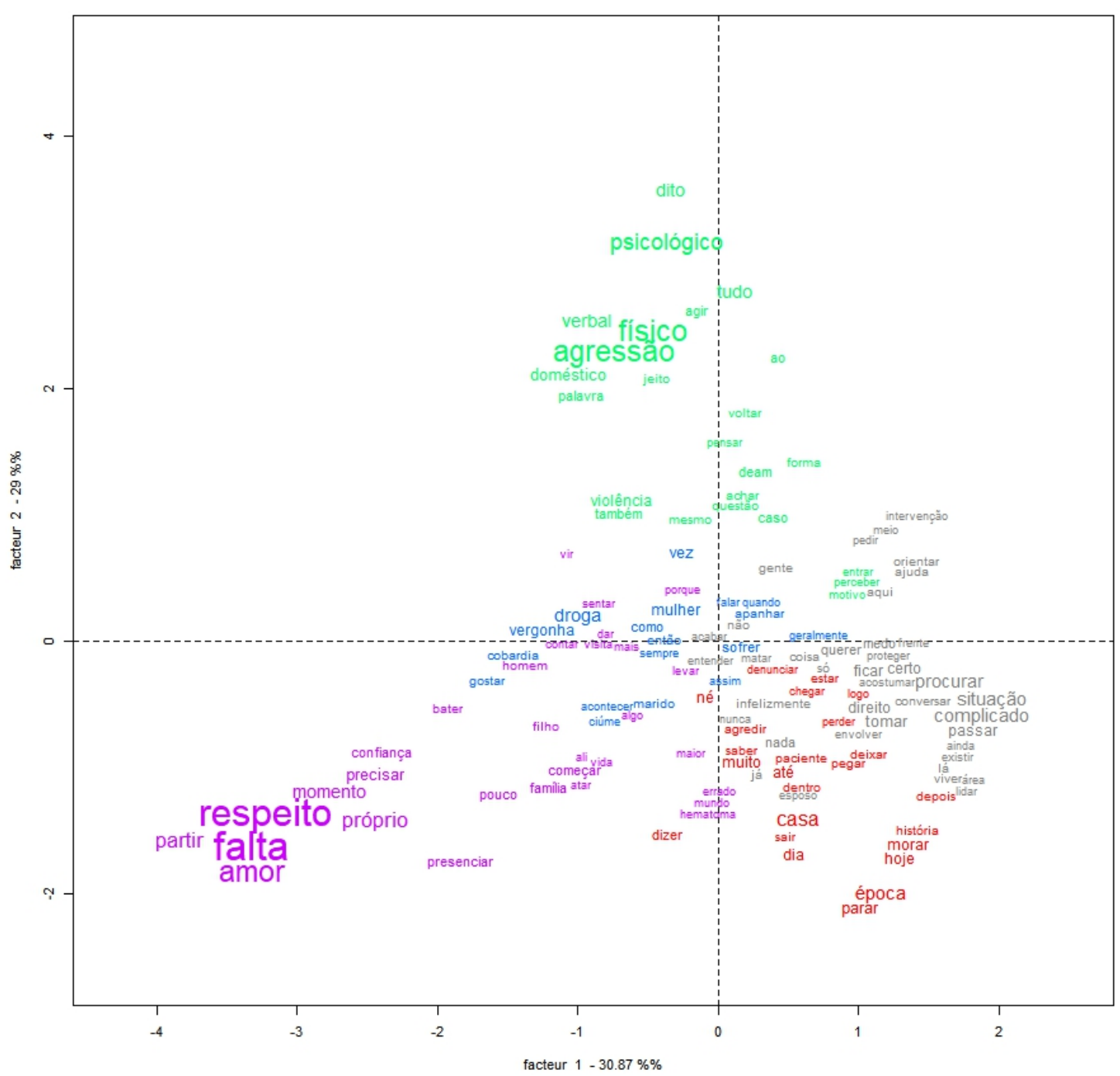

Fonte: Dados da pesquisa, 2017.

[...]O que entendo é que uma agressão, né, que a mulher passa, não só fisicamente como verbalmente (ACS 1).

[...] É todo tipo de agressão que a mulher sofre verbalmente ou fisicamente, trazendo muitos traumas e consequências graves $e$ muitas dessas mulheres procuram ajuda e outras não, elas convivem com o agressor o tempo todo sem procurar nenhuma ajuda (ACS 10)

Já, no segundo quadrante, localizado na parte superior à direita, encontram-se as palavras: DEAM, intervenção, orientar e ajuda. Tais evocações estão relacionadas às atitudes a serem tomadas pelo profissional dentista, ACS,
ASB e por mulheres em situação de violência para o enfrentamento das ocorrências.

Nesse sentido, emergem as seguintes falas do segundo quadrante:

[...] uma forma mais em conjunta ou vai ver se na secretaria tem algum psicólogo que possa estar conversando com ela pra poder estar informando as coisas, pra ver se ela dá a denúncia na DEAM (DENT 11).

[...] Então, primeiramente acho que é identificar os casos suspeitos, se existe realmente de fato esses casos, e trazer depois de identificados, pra ver uma estratégia, uma intervenção (ACS 7). 
[...] Haveria, eu acho que da nossa parte teria uma orientação, orientála a procurar ajuda, eu acho que sim, porque faz parte assim do nosso papel, não só orientar para encaminhamento para a saúde, mas também para a saúde mental, psicológica, ou até ajuda policial (ASB 13).

Referente ao terceiro quadrante, encontrado na parte inferior direita da figura 2, encontraram-se as seguintes evocações: procurar, situação, complicado, direito. Como termos de menor destaque: história, morar, medo. Essas palavras representam as concepções dos entrevistados acerca de mulheres em situação de violência doméstica e como as mesmas reagem ao se depararem nessa situação. A seguir, as falas evidenciadas no terceiro quadrante:

[...] eu acho que as pessoas deveriam se amar mais primeiro ou se conscientizar, a mulher como é o alvo né, procurar o seu valor próprio, se amar mais porque $o$ direito de todo mundo é igual, o homem e mulher tem o direito de viver independente de qualquer coisa (ACS, E4).

[...] Haveria, eu acho que da nossa parte teria uma orientação, orientála a procurar ajuda (ASB, E13).

[...]Eu acho que deveria fazer uma reunião, conversar com a supervisora e dependendo dela ver qual a melhor decisão a ser tomada porque é uma situação que cabe muito cuidado, é muito delicado a forma de lidar com isso porque existe a agressão, mas a gente não pode chegar diretamente e tentar agir de qualquer forma (ACS, E4).

Por fim, no último quadrante localizado na parte inferior esquerda do segundo plano, despontam as seguintes palavras: respeito, falta, amor, próprio. $\mathrm{E}$ como termos de menor repetição, obteve-se: momento, família, marido, covardia. Essas evocações caracterizam o entendimento dos participantes da pesquisa sobre os prováveis motivos que culminam e/ou predispõem as ocorrências dos atos violentos.
[...] É a falta de amor que tem um pelo outro, ou seja, uma falta de respeito um pelo outro ou uma insegurança (ACS, E4).

[...] falta de amor, de respeito, de companheirismo, e eu acho que esse próprio mundo atrapalhado com drogas, esses tipos de coisas que leva a esse tipo de violência né, o álcool, a droga, esse tipo de coisa, eu acho que a gente ver mais nesse ponto aí (TEC-ENF, E18).

[...]A renda baixa, que o homem não quer dividir as despesas com a mulher, ciúmes, falta de confiança, falta de amor, porque se tivesse amor, nada disso ia acontecer muitos maridos folgados também (ACS, E19)

\section{Discussão}

A sociedade tem cada vez mais utilizado de formas violentas para a resolução de conflitos, sinalizando uma falta de abertura para mediações, principalmente no que se direciona a fala, uma vez que é através dela que irá se abrir a possibilidade de negociação, argumentação e assim evitar que formas de violência sejam tidas como a resolução dos problemas ${ }^{11}$.

A VDCM tem se tornado uma realidade no cotidiano de muitas mulheres, no qual fatores sociais, culturais e religiosos estão correlacionados ao fenômeno. Nesse sentido, foi observado que se tornou um problema de saúde pública, considerando que se nota altas taxas de morbidade no Brasil e no mundo ${ }^{12}$. Existem diversas formas de violência, dentre elas a psicológica, física, sexual, patrimonial e moral são as mais conhecidas e vistas nos dia a dia dos serviços de saúde ${ }^{13}$.

Assim, os serviços de Atenção Primária à Saúde (APS) tornam-se um dos locais mais solicitados por mulheres em situação de violência, no entanto, não como primeira demanda ao atendimento a violência, essa surge como uma demanda implícita ${ }^{14}$. Logo, a identificação de casos violentos e também prevenção de possíveis situações de risco à integridade das mulheres é de extrema importância para possibilitar uma boa assistência e resolutividade ${ }^{15}$. Isto pode ser observado durante as entrevistas, falas de ACS e enfermeiras revelaram que mulheres buscavam o atendimento na unidade queixando-se de fortes dores ou apresentando edemas, mas sem 
descrever como surgiram as lesões ou, muitas vezes, se contradizendo na descrição dos fatos, na tentativa de omitir o ocorrido, seja por vergonha, medo ou crença de que foi um fato isolado.

Observa-se que a RS dos profissionais que atuam na rede primária de atenção à saúde contém elementos ainda entrelaçados ao uso de drogas, desemprego, falta de diálogo, demonstrando a influência sociocomunitária adquirida no local de trabalho quando se conceitua violência ${ }^{16}$.

Ainda, as mesmas também não buscam apoio nas Delegacias Especializadas no Atendimento à Mulher (DEAM), criadas pela Polícia Civil, que propõe e realiza ações de prevenção, proteção e investiga crimes de violências contra mulheres ${ }^{17}$.

É observado que ainda hoje, mesmo com tantas campanhas a respeito do tema, as mulheres em situação de violência encontram dificuldades ou resistem à busca de ajuda para a violência sofrida, devido suas Representações Sociais seja por vergonha, medo ou dificuldade em expor o ocorrido por receio de julgamentos. O atendimento no âmbito da saúde é direcionado as queixas principais referidas pelas mulheres, e esse entendimento está fixando tanto nos que buscam o atendimento quanto nos profissionais, não vendo a unidade de saúde como um local de busca ativa de ajuda, mas apenas, como ambiente curativo ${ }^{18}$.

Por parte da mulher em situação de violência e nesse momento a solicitante do atendimento em saúde, deve-se ao receio de dizer algo que possa gerar mais conflito com o parceiro ou ainda entender que esta é uma situação a ser resolvida entre o casal, bem como a esperança de que este seja um caso que não voltará a se repetir. Já, no que se refere aos profissionais de saúde, por vezes a alta demanda dos atendimentos de rotina levam a tentar otimizar o seu tempo e buscam tratar e acompanhar apenas a queixa principal da mulher, sem se atentar a descobrir o fator gerador das lesões ${ }^{19}$.

A agressão física é ainda a mais conhecida e engloba toda forma de ato violento que cause algum dano físico à pessoa em situação de violência. Essa forma é a mais conhecida entres os profissionais de saúde, pois as mulheres chegam ao serviço e são encontradas com marcas físicas pelo corpo ${ }^{20}$. Dessa maneira, foi observada que uma das palavras mais evocadas foi agressão, corroborando com os estudos que indicam que os profissionais dos profissionais da saúde que trabalham nas USF contêm elementos ligados à atividade profissional em ambientes ambulatoriais e hospitalares, visto que o significado de agressão física está relacionado à assistência para mulheres em situação de violência ${ }^{21}$. É notado o sentido dessa informação ao se analisar que os profissionais da rede da APS possuem concepções do uso de drogas, o desemprego, a falta de diálogo, correlacionados à violência ${ }^{22}$.

No plano cartesiano é visto elementos que caracterizam a vergonha, que pode caracterizar uma atitude das mulheres diante da agressão sofrida. Percebe-se que há uma maior compreensão acerca das ações que caracterizam a violência.

A notificação da violência contra a mulher é obrigatória em todo o Brasil devendo ser realizada pelo profissional de saúde responsável pela pessoa em situação de violência, entretanto, tal ação ainda não está sendo corretamente executada ${ }^{23}$. Isso se deve ao fato de existir lacunas no conhecimento dos profissionais, considerando que ainda não se é trabalhada a notificação de casos, bem como a abordagem com essas mulheres. Logo, toda e qualquer busca acerca de consultas em unidades de saúde por mulheres nessa situação, devem ser aproveitadas para se tornar um espaço de troca e aprendizado, onde as mesmas possam, de fato, relatar o acontecido sem medo ou receio, se sentido seguras nesse momento ${ }^{24}$.

Achados na literatura demonstram que raramente a violência física ocorre de maneira isolada, dessa forma, é enfatizada a necessidade de reconhecimento da violência sofrida por mulheres também em outros contextos como psicológicas, patrimonial, moral, sexual, e assim possam preveni-la, bem como disponibilizarem cuidados a essas mulheres, através de rodas de conversas, panfletagem, diálogos propostos por ACS em suas áreas ${ }^{25}$. Partindo dessa análise, é importante que os profissionais da saúde, por estarem no primeiro nível de atenção à saúde, conhecer e se apropriar, através de estudos que abordem outras formas de violência, para que possam ajudar de uma maneira mais efetiva nessa questão.

O estudo possui limitações, uma vez que foi realizado em um município do Estado da Bahia em equipes da zona urbana, requerendo a abrangência pra a zona rural e outras localidades. Além disso, observou-se a necessidade de realizar mais estudos sobre o preparo dos profissionais para o enfretamento da violência doméstica contra a mulher. 


\section{Conclusão}

O estudo mostrou que a violência doméstica conta a mulher é bem conhecida entre os profissionais da atenção básica, uma vez que os mesmos sabem conceituá-la e caracterizá-la, entretanto, ainda se faz necessário melhores abordagens dos profissionais com as mulheres em situação de violência, de modo que durante os atendimentos, os mesmos consigam identificar melhor casos de violência para que a mulher se sinta mais acolhida e não tenha medo de procurar ajuda.

É necessário que exista uma rede de apoio que possibilite o cuidado inicial, a comunicação com os familiares dessa mulher, bem como o encaminhamento da mesma para os órgãos responsáveis e necessários para a resolução da questão. Além disso, se faz necessário a solicitação de serviços que aborde todas as demandas aos cuidados emergenciais dessas mulheres ao chegarem ao serviço.

Estudos nessa visão não acabam com a necessidade de mais pesquisas sobre o tema, mas permite instigar questionamentos sobre a organização dos serviços de saúde e a percepção destes profissionais sobre a VDCM.

\section{Referências}

1. Silva CD, et al. Representação social da violência doméstica contra a mulher entre técnicos de enfermagem e agentes comunitários. Revista da Escola de Enfermagem da USP. São Paulo. 2015; 49(1): 22-9.

2. Guimarães $M C$, Pedroza RLS. Violência contra a mulher: problematizando definições teóricas, filosóficas e jurídicas. Psicologia \& Sociedade. 2015; 27(2): 256-266.

3. Cerqueira D, Lima RS, Bueno S, Neme C, Ferreira H, Alves PP. Atlas da violência 2019. Brasília: Rio de Janeiro: São Paulo: Instituto de Pesquisa Econômica Aplicada; Fórum Brasileiro de Segurança Pública, 2019.

4. Brasil. Secretaria de Políticas para as Mulheres. Lei Maria da Penha: Lei 11.340 de 07 de agosto de 2006 - Coíbe a violência doméstica e intrafamiliar contra a mulher. Brasília. Presidência da República; 2018.

5. Rosa DOA, Ramos RCS, Gomes TMV, Melo EM, Melo VH. Violência provocada pelo parceiro íntimo entre usuárias da Atenção Primária à Saúde: prevalência e fatores associados. Saúde debate. Rio de Janeiro. 2018; 42(4).

6. Nascimento ARA, Menandro PRM. Análise lexical e análise de conteúdo: uma proposta de utilização conjugada. Estudos e Pesquisas em Psicologia. 2006; 6(2):72-8

7 Correia CM, Gomes NP, Couto TM, Rodrigues AD, Erdmann AL, Diniz NMF, et al. Representações sobre o suicídio para mulheres com história de violência doméstica e tentativa do mesmo. Texto \& Contexto Enferm 2014; 23: 118-25

8. Moscovici S. Representações sociais: investigações em psicologia social. 9a ed. Petrópolis, RJ: Vozes; 2012.

9. Bardin L. Análise de conteúdo. 3a reimpressão da 1ạ ed de 2011. São Paulo: Edições 70, 2011; 123-31

10. Ministério da Saúde. Portaria n. 2.436, de 21 de setembro de 2017. Aprova a Política Nacional de Atenção Básica, estabelecendo a revisão de diretrizes e normas para a organização da Atenção Básica, no âmbito do Sistema Único de Saúde. Diário Oficial da União. Brasília; 2017

11. Oliveira JM. A violência doméstica e familiar contra a mulher - poder e gerações sociais. Diálogos Possíveis. 2016; 15(2):127-47

12. Rosal DOAR, Ramos RCS, Gomes TMV, Melo EM, Melo VH. Violência provocada pelo parceiro íntimo entre usuárias da Atenção Primária à Saúde: prevalência e fatores associados. Rio de Janeiro. 2018; 42(4): 67-80

13. Stockman JK, Lucea MB, Bolyard R, Bertand D, Callwood GB, Sharps PW, et al. Intimate partner violence among African American and African Caribbean women: prevalence, risk factors, and the influence of cultural attitudes. Glob Health Action. 2014; $7: 24772$

14. Bozzo ACB, Matos GC, Beraldi LP, Souza MD. Violência doméstica contra a mulher: caracterização dos casos notificados em um município do interior paulista. Revist Enferm UERJ. 2017; 25: e11173: 1-5

15. Sweet, PL. 'Every bone of my body:' Domestic violence and the diagnostic body. Soc Sci Med. 2014; 122: 44-52.e. DOI: https://doi.org/10.1016/j.socscimed.2014.10.014

16. García-Moreno C, Hegarty K, Oliveira AFL, Koziol-McLain J, Colombini M, Feder G. The health-systems response to violence against women. The Lancet. 2015 Apr; 385(9977): 156779. Available from: http://www.thelancet.com/pdfs/journals/l ancet/PIIS0140-6736(14)61837-7.pdf.

17. Souza L, Cortez MB. A delegacia da mulher perante as normas e leis para o enfrentamento da violência contra a mulher: um estudo de caso. Rev. Adm. Pública. 2014; 48(3)

18. Guedes RN, Fonseca RMGS, Egry EY. Limites e possibilidades avaliativas da estratégia 
saúde da família para a violência de gênero. Rev. Esc. Enfermagem USP. 2013; 47(2):304-11.

19. Vives-Cases C, Ruiz-Cantero MT, Aguir $V E$, et al. The effect of intimate partner violence and other forms of violence against women on health. Journal of Public Health. 2010; 33(1):1521

20. Gomes NP, Silveira YM, Diniz NMF, Paixão GPN, Camargo CL, Gomes NR. Identificação da violência na relação conjugal a partir da Estratégia Saúde da Família. Texto Contexto Enferm. 2013; 22(3):789-96.

21. Nascimento EFGA, Ribeiro AP, Souza ER. Percepções e práticas de profissionais de saúde de Angola sobre a violência contra a mulher na relação conjugal. Cad Saúde Pública. 2014; 30(6):1-10.

22. Santi LN, Nakano AMS, Lettiere A. Percepção de mulheres em situação de violência sobre o suporte e apoio recebido em seu contexto social. Texto Contexto Enferm. 2010; 19(3):417-24.

23. Santos MFS. Representações sociais e psicologia social. In: Almeida AMO, Jodelet D. Representações sociais: interdisciplinaridade e diversidade de paradigmas. Brasília, DF: Thesaurus, 2009.

24. Brasil. Lei no 10.778 , de 24 de novembro de 2003. Estabelece a notificação compulsória, no território nacional, do caso de violência contra a mulher que for atendida em serviços de saúde públicos ou privados. Diário Oficial da União; 2003.

25. Silva CD, Gomes VLO, Fonseca $A D$, Gomes MT, Arejano TGCB. Representação da violência doméstica contra a mulher: comparação entre discentes de enfermagem. Rev Gaúcha Enferm. 2018; 39:e63935.

\section{Endereço para Correspondência}

Universidade Estadual do Sudoeste da Bahia (UESB)

Av. José Moreira Sobrinho, s/n - Jequiezinho Jequié (BA)

CEP.: 45.205-490

e-mail: vprodrigues@uesb.edu.br

Recebido em 06/07/2020

Aprovado em 09/09/2020

Publicado em 20/01/2021 